\title{
Acamprosate for treatment of alcohol dependence: mechanisms, efficacy, and clinical utility
}

This article was published in the following Dove Press journal:

Therapeutics and Clinical Risk Management

31 January 2012

Number of times this article has been viewed

\author{
Katie Witkiewitz \\ Kimber Saville \\ Kacie Hamreus \\ Department of Psychology, \\ Washington State University \\ Vancouver, Vancouver, WA, USA
}

Correspondence: Katie Witkiewitz Department of Psychology, Washington State University Vancouver, I4204 NE Salmon Creek Ave, Vancouver, WA 98686, USA

$\mathrm{Tel}+\mathrm{I} 3605469403$

$\mathrm{Fax}+\mathrm{I} 3605469038$

Email katie.witkiewitz@vancouver.wsu.edu
Abstract: Acamprosate, or $N$-acetyl homotaurine, is an $N$-methyl-D-aspartate receptor modulator approved by the Food and Drug Administration (FDA) as a pharmacological treatment for alcohol dependence. The exact mechanism of action of acamprosate is still under investigation, but the drug appears to work by promoting a balance between the excitatory and inhibitory neurotransmitters, glutamate and gamma-aminobutyric acid, respectively, and it may help individuals with alcohol dependence by reducing withdrawal-associated distress. Acamprosate has low bioavailability, but also has an excellent tolerability and safety profile. In comparison with naltrexone and disulfiram, which are the other FDA-approved treatments for alcohol dependence, acamprosate is unique in that it is not metabolized by the liver and is also not impacted by alcohol use, so can be administered to patients with hepatitis or liver disease (a common comorbid condition among individuals with alcohol dependence) and to patients who continue drinking alcohol. Acamprosate has demonstrated its efficacy in more than 25 placebocontrolled, double-blind trials for individuals with alcohol dependence, and has generally been found to be more efficacious than placebo in significantly reducing the risk of returning to any drinking and increasing the cumulative duration of abstinence. However, acamprosate appears to be no more efficacious than placebo in reducing heavy drinking days. Numerous trials have found that acamprosate is not significantly more efficacious than naltrexone or disulfiram, and the efficacy of acamprosate does not appear to be improved by combining acamprosate with other active medications (eg, naltrexone) or with psychosocial treatment (eg, cognitive-behavioral therapy). In this review, we present the data on acamprosate, including its pharmacology, efficacy, safety, and tolerability in the treatment of alcohol dependence.

Keywords: alcohol abuse, acamprosate, $N$-methyl-D-aspartate receptor, gammaaminobutyric acid

\section{Management issues in alcohol dependence}

The harmful use of alcohol is a global public health problem, with nearly 2.5 million deaths per year attributed to alcohol worldwide. The World Health Organization ${ }^{1}$ has identified alcohol as the first and second leading risk factor for premature mortality and disability in the Americas and Europe, respectively. Alcohol dependence is one of the most common substance use disorders, with more than $12.5 \%$ of individuals in the US (roughly 38 million) meeting criteria for alcohol dependence within their lifetime and 3.8\% (roughly 11 million) meeting criteria in the past year, ${ }^{2}$ with similar rates in many European countries. ${ }^{3}$

Alcohol dependence has been described as a chronic, relapsing disorder, ${ }^{4}$ with the majority of individuals never receiving treatment. One recent survey found that fewer 
than $15 \%$ of individuals with a lifetime diagnosis of alcohol abuse or dependence have ever received treatment for alcohol dependence, ${ }^{5}$ and of those who do seek treatment, fewer than $13 \%$ are provided with prescription medication for alcohol dependence. ${ }^{6}$ The majority of individuals with disorders related to alcohol use receive psychosocial intervention, such as cognitive-behavioral treatment, coping skills training, contingency management, motivation enhancement therapy, and mutual support groups (eg, Alcoholics Anonymous). Overall, most psychosocial interventions, including self-help, have been found to be effective in the treatment of alcohol dependence. ${ }^{7}$ However, the majority of patients experience a lapse within the first year following a psychosocial intervention, ${ }^{8,9}$ and there is growing evidence that combining psychosocial and pharmacological treatments may be an effective method for treating alcohol dependence. ${ }^{10-12}$

Currently, there are three medications for the treatment of alcohol dependence that have been approved by the US Food and Drug Administration (FDA), ie, disulfiram, naltrexone, and acamprosate. Disulfiram blocks the metabolism of acetaldehyde, which leads to severe discomfort when alcohol is consumed. Disulfiram has been approved for the treatment of alcohol dependence for over 50 years and has been shown to improve rates of alcohol abstinence, ${ }^{13}$ but numerous disadvantages of disulfiram have been identified. Namely, disulfiram is an aversion therapy and is only effective when patient compliance is high, so the continued use of disulfiram requires a significant level of patient motivation. Naltrexone is an opioid receptor antagonist that was approved by the FDA in 1994 as an oral medication, and a long-acting injectable formulation was approved in 2006. Naltrexone appears to work by competitively binding to endogenous opioid receptors and reducing the pleasant and reinforcing effects of alcohol. Unlike disulfiram, which relies on aversion, naltrexone directly targets the learned association of experiencing euphoria following alcohol use. A recent meta-analysis of 50 randomized controlled trials of naltrexone in the treatment of 7793 patients with alcohol dependence found that naltrexone reduced the risk of heavy drinking days by $83 \%$ and decreased the number of drinking days by $4 \%$ in comparison with placebo. ${ }^{14}$ Numerous reviews of naltrexone have also found varying levels of responsiveness to the medication, which may be explained by a polymorphism in the OPRM1 gene (a $\mu$-opioid receptor gene) ${ }^{15}$ although a recent prospective study of naltrexone in combination with cognitive-behavioral therapy failed to find an effect of genotype on response to treatment with naltrexone. ${ }^{16}$ The primary side effects of naltrexone include gastrointestinal problems (eg, abdominal pain, nausea), symptoms associated with decreased central nervous system arousal (eg, daytime sleepiness, fatigue), blurred vision, decreased libido, depression, and dizziness. ${ }^{14}$

Acamprosate, marketed in the US under the brand name

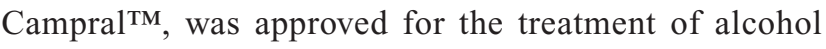
dependence in France in 1989 and by the FDA in 2004. As described in more detail below, acamprosate is thought to promote a balance between the excitatory and inhibitory neurotransmitters, glutamate and gamma-aminobutyric acid (GABA), respectively, and may help individuals with alcohol dependence by modifying learned responses to alcohol cues and by reducing the distress associated with withdrawal. There is also some evidence that acamprosate may have neuromodulatory and neuroprotective effects. ${ }^{17,18}$ In this review, we present the data on acamprosate, including a brief overview of its pharmacology and a review of the efficacy, safety, and tolerability of acamprosate in the treatment of alcohol dependence.

\section{Data sources}

A literature review using the PubMed and EMBASE databases with the search terms "acamprosate" and "alcohol dependence" or "alcohol use disorder" or "alcoholism" was conducted to find relevant articles published in English. Over 2000 references were identified. We also reviewed completed and ongoing clinical trials by searching http://clinicaltrials. gov and http://projectreporter.nih.gov. All searches were last updated on January 12, 2011.

\section{Pharmacology, mode of action, and pharmacokinetics of acamprosate}

The structure of acamprosate ( $N$-acetyl homotaurine; 3-(acetylamino)-1-propanesulfonic acid calcium salt) is similar to that of several neurotransmitters, both excitatory and inhibitory, and helps balance adaptations in neuronal firing from chronic alcohol consumption by targeting both $N$-methyl-D-aspartate (NMDA) glutamatergic and GABA receptor activity. ${ }^{19}$

Due to its structural similarity to GABA and taurine, the potential for acamprosate to act on inhibitory receptors was an early focus, ${ }^{20}$ but more recent research indicates little influence of acamprosate on $\mathrm{GABA}_{\mathrm{A}}$ or glycine receptors, either independently or in the presence of ethanol, ${ }^{21}$ and current efforts have shifted toward examining the mechanism of action of acamprosate at NMDA receptors.

Acamprosate appears to act as a partial coagonist of NMDA receptors, depending on its concentration and the 
activity of the NMDA receptor, where at low concentrations acamprosate increases low receptor activity and at high concentrations inhibits highly activated NMDA receptors. ${ }^{22}$ The precise mode of action is not fully understood, but recent studies suggest that acamprosate is an NMDA receptor modulator ${ }^{17}$ rather than a direct antagonist, potentially working via a spermidine-sensitive binding site. ${ }^{22}$ Upon further examination, acamprosate may be responsible for regulating NMDA subunit synthesis, indicating an important role in amending the changes caused by chronic alcohol consumption rather than acutely antagonizing NMDA-mediated glutamatergic neurotransmission. ${ }^{23}$ Thus, acamprosate helps balance disrupted neurotransmission, mainly by decreasing the overexcitation caused by alcohol dependence. Acamprosate is most likely absorbed via a paracellular route ${ }^{24}$ and overall bioavailability is relatively low (11\%). ${ }^{25}$ Maximum plasma concentrations were observed to range from 4.3-15.3 hours after acute oral administration of varying formulas but dropped to 3.5-9.5 hours when steady state was reached. ${ }^{25}$ A steady state has consistently been observed between 5 and 7 days. ${ }^{25,26}$ Limited absorption after oral administration results in a majority of the drug being excreted in fecal matter, ${ }^{25}$ so amounts excreted in urine may be directly correlated with its bioavailability. ${ }^{24}$ Acamprosate is not processed significantly in the liver and does not appear to have any metabolites, leaving little threat of interactions with other drugs, including naltrexone, ${ }^{27}$ disulfiram, diazepam, or alcohol, but taking food with acamprosate does appear to limit its absorption even further. ${ }^{25}$

\section{Efficacy in alcohol dependence}

The efficacy of acamprosate in the treatment of alcohol dependence was recently evaluated in a comprehensive Cochrane review that included 24 randomized controlled trials of 6894 patients with the disorder. The 24 randomized controlled trials included in the Cochrane review all used a double-blind design to compare the effects of acamprosate (alone or in combination) with placebo or an active treatment control group. The primary outcomes of the review included returning to any drinking and cumulative duration of abstinence (ie, the sum of days a patient remained abstinent during the study). The secondary outcomes included return to heavy drinking (defined in most studies as five or more standard drinks per occasion), liver enzyme levels (gammaglutamyl transpeptidase), and side effects. A meta-analysis of the 24 trials indicated a statistically significant effect of acamprosate in comparison with placebo on both of the primary outcomes. Receiving acamprosate significantly reduced the risk of returning to any drinking by $86 \%$ (95\% confidence interval [CI]: 81\%-91\%) and increased the cumulative duration of abstinence by $11 \%$ (95\% CI: 5\%-16\%) compared with placebo during treatment. A statistically significant difference between groups was also observed 3-12 months after treatment was discontinued. Individuals who received acamprosate had a $9 \%$ lower risk of returning to any drinking and a 9\% higher continuous abstinence duration in comparison with placebo after discontinuing treatment. The Cochrane review did not find significant differences between the acamprosate and placebo groups on return to heavy drinking or on gamma-glutamyl transpeptidase, and the review also did not find significant differences between acamprosate and naltrexone on the primary outcomes or return to heavy drinking. There was a significant difference between acamprosate and naltrexone on gamma-glutamyl transpeptidase. Likewise, the combination of acamprosate and naltrexone did not lead to significant differences in primary or secondary outcomes, as compared with placebo or as compared with acamprosate monotherapy.

The Cochrane review and other reviews of acamprosate ${ }^{18,28,29}$ have generally omitted open-label and single-blind studies because they often lack the rigorous standards of doubleblind, randomized trials and can be lower in internal validity. It is also the case that double-blind, placebo-controlled, randomized trials are sometimes lower in external validity. For this reason, we believe that it is important to consider the findings from less methodologically rigorous evaluations of acamprosate in addition to the review of the double-blind, placebo-controlled trials described above. The Cochrane review excluded 24 trials of acamprosate in the treatment of alcohol dependence for a variety of reasons, including openlabel or single-blind design, lack of placebo or active control groups, treatment duration below 30 days, or follow-up duration less than 8 weeks, and nonavailability of data. Of these 24 trials, we provide an overview of the findings from nine open-label and single-blind studies in Table 1.

As seen in Table 1, acamprosate was less effective than naltrexone ${ }^{30,31}$ and disulfiram, ${ }^{31,32}$ was more effective than placebo or standard care,,$^{33,34}$ and was not significantly different from oxcarbazepine. ${ }^{35}$ Overall, the average number of days until the first drinking day among those who received acamprosate ranged from $66^{35}$ to 81.5 days, ${ }^{36}$ while the average days until the first drinking day among the comparison groups ranged from 96 days for oxcarbazepine ${ }^{35}$ to 123 days for disulfiram. ${ }^{32}$ The average number of days to a heavy drinking day or severe lapse among those who received acamprosate ranged from 17.6 days $^{31}$ to 77 days, ${ }^{35}$ while 

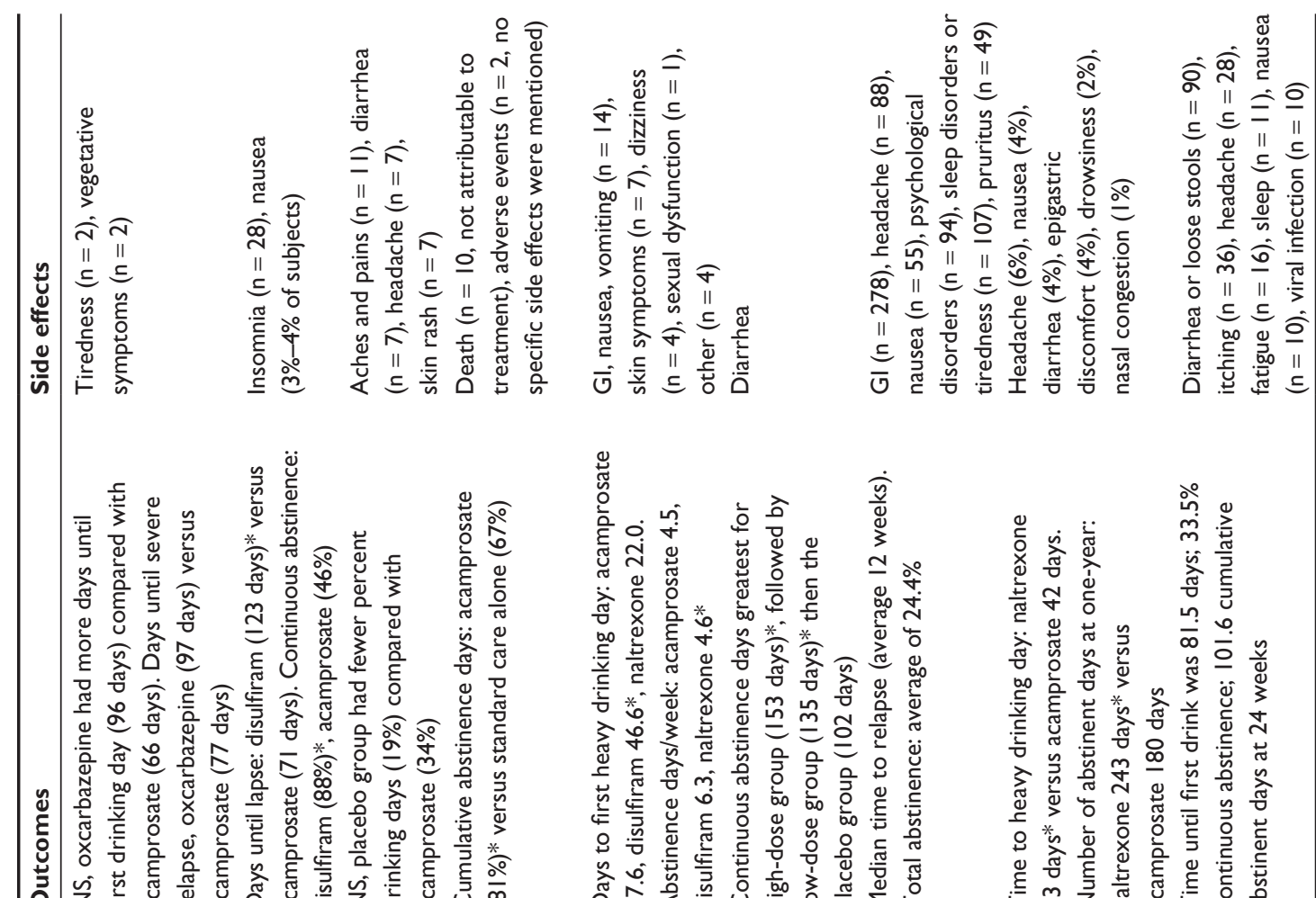

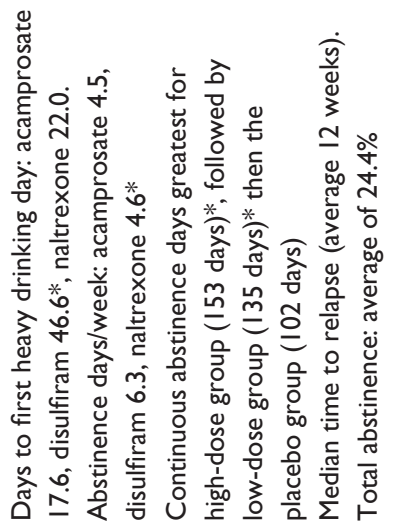

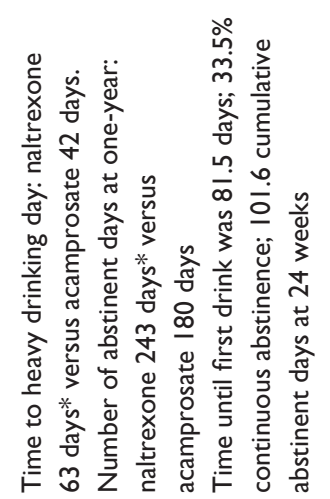

= 웅

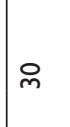

$$
\text { 의 } \quad \text { }
$$

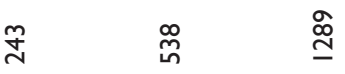

$\stackrel{\infty}{\underline{n}}$

n
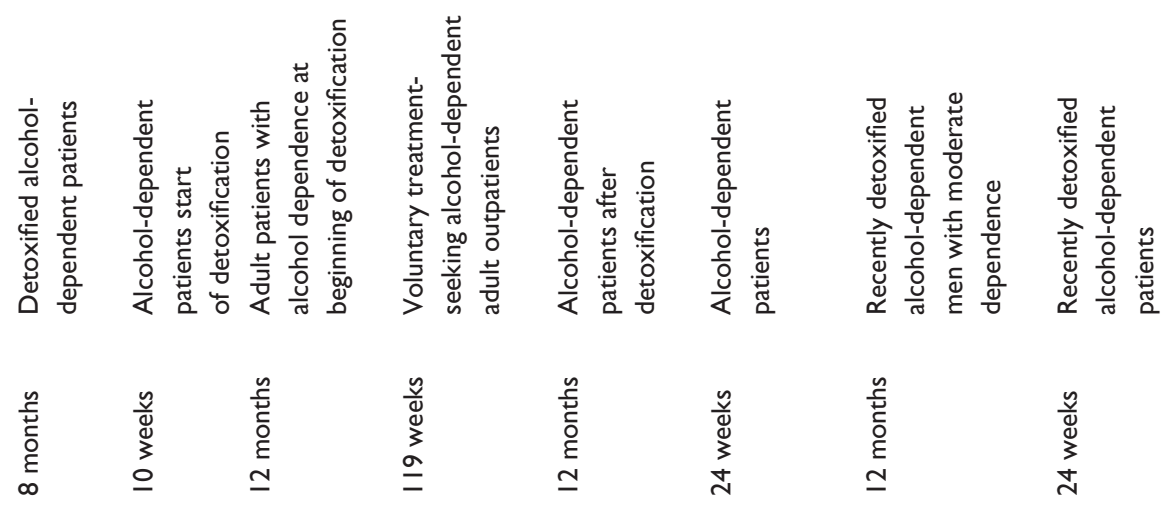

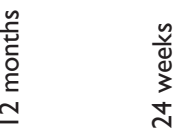
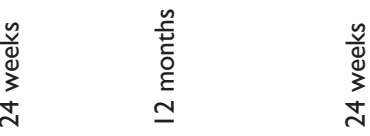

言

要

ते ते

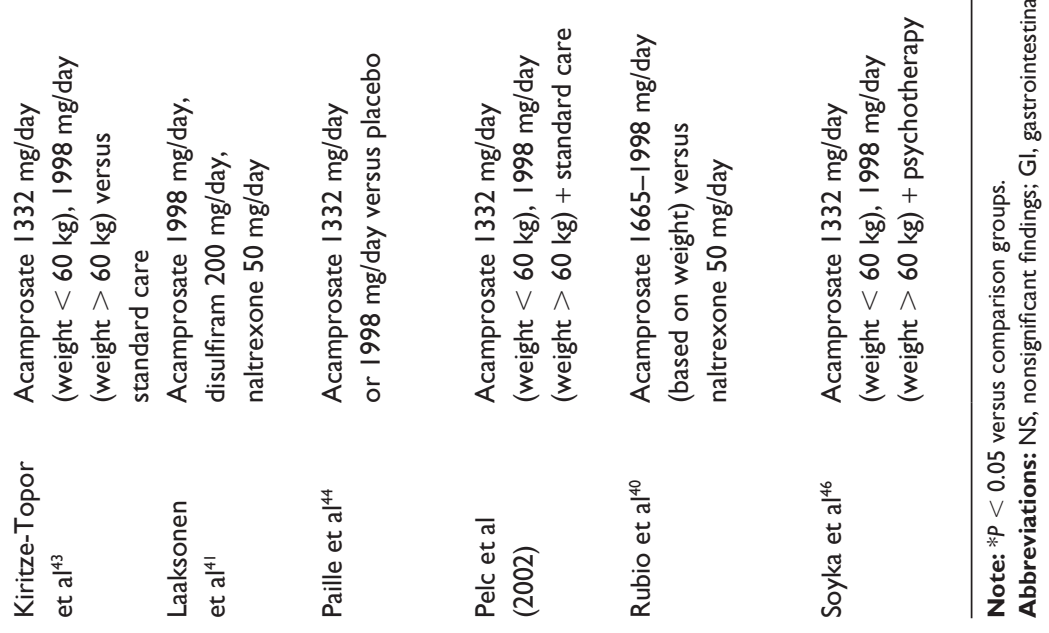


the average days until the first heavy drinking day or severe lapse among the comparison groups ranged from 22 days for naltrexone $^{31}$ to 97 days for oxcarbazepine. ${ }^{35}$

Additional randomized controlled trials have been published since the Cochrane review was completed, with the latest studies included in the Cochrane review published in 2006. To address this gap we will briefly summarize each of the randomized trials that have been published since 2006. Most recently, acamprosate was combined with either integrative behavioral therapy or supportive counseling, and was compared with integrative behavioral therapy and placebo in a randomized, double-blind multisite study. ${ }^{12}$ A total of 371 individuals were randomized to one of the three treatments (acamprosate + behavioral therapy, acamprosate + supportive counseling, or placebo + behavioral therapy) administered for 6 months, and patients were followed for 3 and 6 months after discontinuation of treatment. Importantly, individuals were dropped from the study if they used any alcohol during treatment (40.3\% of 201 dropouts) or missed three consecutive sessions (35.3\% of 201 dropouts). At the end of the 6-month treatment and up to 6 months following treatment, there were no significant differences in success (nonlapsed) rates between the three groups. The best outcomes were observed in the acamprosate + behavioral therapy condition (47.6\% success rate at end of treatment and $35 \%$ success rate at 6-month follow-up) and the placebo + behavioral therapy condition $(48 \%$ success rate at end of treatment and $32 \%$ success rate at 6-month follow-up), as compared with the acamprosate + supportive counseling condition (37.7\% success rate at end of treatment and 32\% success rate at 6-month follow-up). Other drinking outcomes (eg, rates of moderate, nonheavy drinking) were not reported.

\section{Compliance and patient response}

Importantly, compliance with medication plays an important role in the efficacy of acamprosate. One recent meta-analysis of individual patient data $(n=2305)$ from 11 randomized controlled trials comparing acamprosate with placebo found that those individuals who took at least $80 \%$ of their prescribed medication during the early phases of treatment were five times more likely to complete treatment and had a significantly higher percentage of abstinence days than those individuals who were not compliant. ${ }^{37}$ The authors also found a significant compliance by treatment interaction in the prediction of abstinence days. Individuals who complied with acamprosate had $8.4 \%$ more improvement in percentage of abstinent days than individuals who complied with placebo. Those individuals who complied with acamprosate
$50 \%$ of the time had only a $4.2 \%$ improvement in percentage of abstinent days as compared with those individuals who complied with placebo. These findings are noteworthy given the three times daily dosing and unpleasant side effects of acamprosate (eg, nausea, diarrhea), which may cause disruptions in compliance. In the largest randomized clinical trial of acamprosate conducted to date, ${ }^{10}$ mean adherence to acamprosate monotherapy was only $69.7 \%$ and mean adherence to acamprosate combined with naltrexone was only $64.9 \%$.

Genetic factors may also play a role in the patient response to acamprosate. Kiefer et $\mathrm{al}^{38}$ found that genetic variations in a gene for GATA-binding protein (GATA4) was significantly associated with relapse risk during a 90-day medical treatment period and that the risk was primarily driven by those patients who were treated with acamprosate as compared with those treated with naltrexone or placebo. Similarly, in a novel study of genetic risk factors interacting with either naltrexone or acamprosate in the prediction of alcohol cue reactivity, Ooteman et $\mathrm{al}^{39}$ found interactions between medication condition and polymorphisms of the GABRA6 and $D R D 2$ genotypes in prediction of cue-induced craving. The efficacy of acamprosate on cue-induced craving depended on the frequency of the $\mathrm{C}$ allele of the GABRA6 gene. For the $D R D 2$ gene, acamprosate outperformed naltrexone among A1 allele carriers and naltrexone outperformed acamprosate among A2 allele carriers. The genetic findings from these two studies need to be replicated prior to making any definite conclusion, but the results are promising for future pharmacogenetic research on the efficacy of matching acamprosate to profiles of genetic risk.

\section{Safety and tolerability}

Acamprosate has been described as having an "excellent safety profile". ${ }^{40}$ Perhaps most important for the treatment of alcohol dependence, acamprosate is not metabolized by liver enzymes and alcohol has no effect on the pharmacokinetics of acamprosate. Acamprosate is excreted through the kidneys and is contraindicated for individuals with severe renal impairment. A systematic review of the published literature from 1990 to 2002 found that patients who received acamprosate generally reported few adverse effects and also reported fewer adverse effects than those patients who received naltrexone. ${ }^{41}$ In the Cochrane review, only diarrhea was reported significantly more often for acamprosate than for placebo, but the dropout rate due to side effects was $35 \%$ higher in the acamprosate group than in the placebo group. Other side effects reported 
in the studies reviewed by the Cochrane group included abdominal pain (three studies), constipation (three studies), nausea (five studies), vomiting (three studies), other gastrointestinal symptoms (eight studies), pruritus (four studies), and a variety of side effects were reported in one or two of the studies reviewed.

Rosenthal et $\mathrm{al}^{42}$ conducted an analysis of acamprosate safety and tolerability data pooled from 13 large randomized, double-blind, placebo-controlled trials of acamprosate for alcohol dependence. Their analysis included 2272 patients who received acamprosate doses of 1998-2000 mg/day $(\mathrm{n}=1749), 1132 \mathrm{mg} /$ day $(\mathrm{n}=440)$, and $3000 \mathrm{mg} /$ day $(\mathrm{n}=83)$ for an average of 12.7-34.7 weeks. The overall incidence of adverse events pooled across the acamprosate groups was $61 \%$ (as compared with $56 \%$ in the pooled placebo groups). Dropout due to adverse events was not significantly different between the pooled acamprosate (7\% in shorter trials, $8 \%$ in longer trials) and pooled placebo groups (6\% in shorter trials, $7 \%$ in longer trials). Consistent with the data from the Cochrane review, diarrhea was the most common adverse event and was reported significantly more often $(P<0.01)$ by acamprosate patients $(16 \%)$ than by placebo patients $(10 \%)$. The patients reported only mild or moderate diarrhea, and the incidence of diarrhea decreased after 4 weeks. Serious adverse events (defined as any event that was fatal, lifethreatening, or resulted in prolonged hospitalization, disability/incapacity, birth defect, cancer, or overdose) were slightly more common among the acamprosate groups ( $7 \%$ in shorter trials, $14 \%$ in longer trials), as compared with the placebo groups ( $5 \%$ in shorter trials, $11 \%$ in longer trials), although no significant differences in serious adverse events were found in a between-group analysis of the pooled acamprosate versus pooled placebo data.

Overall, numerous narrative and meta-analytic reviews have concluded that acamprosate is safe and well tolerated by patients with alcohol dependence. ${ }^{51,52,40-43}$ Notably, of the three FDA-approved medications for alcohol dependence, acamprosate is the only medication that is not associated with liver toxicity (naltrexone is contraindicated in patients with hepatitis or liver failure) and is also not impacted by alcohol use (disulfiram and alcohol consumption results in significant physical symptoms). In a risk-benefit analysis comparing double-blind, placebo-controlled trials of acamprosate and/or naltrexone in the treatment of alcohol dependence, Mason ${ }^{44}$ concluded that acamprosate is more reliably effective and is often better tolerated, which is consistent with later reviews that found acamprosate to be associated with fewer adverse events in comparison with naltrexone. ${ }^{41}$

\section{Acamprosate in clinical practice}

Based on the available evidence and reviews of acamprosate conducted to date, we conclude that acamprosate is an efficacious treatment for alcohol dependence and is well tolerated by patients. Acamprosate also has no abuse potential and does not have significant drug interactions with many of the medications that are commonly used to treat alcohol dependence and other psychiatric disorders (including naltrexone, antidepressants, anxiolytics, and hypnotics). ${ }^{42}$ Few serious adverse events associated with acamprosate have been reported across studies and fewer than $8 \%$ of patients have dropped out of treatment because of the side effects of acamprosate, ${ }^{42}$ which is lower than the $10 \%$ rate of dropout due to the side effects of naltrexone. ${ }^{41}$

The question of whether acamprosate is a cost-effective treatment was recently evaluated in a systematic review of economic evaluations of pharmacotherapy in the treatment of alcohol dependence. Although the number of studies identified for the review was limited to only seven trials, the results clearly indicated a net benefit of acamprosate, naltrexone, or their combination. Cost-effectiveness of the COMBINE (Combined Pharmacotherapies and Behavioral Interventions for Alcohol Dependence) study, the largest randomized controlled trial of acamprosate conducted to date, ${ }^{45}$ found that naltrexone or the combination of naltrexone with acamprosate produced the greatest cost savings with respect to their effectiveness in increasing percent days of abstinence and increasing the proportion of patients who avoided heavy drinking and were determined to have good clinical outcomes. ${ }^{46} \mathrm{~A}$ second analysis of the COMBINE study evaluated the cost-effectiveness of each treatment condition from the patient's perspective by calculating total costs, effectiveness, and the patient time costs (eg, time spent traveling to and from treatment). ${ }^{47}$ Again, the results indicated that placebo, naltrexone, or the combination of naltrexone and acamprosate, were the most cost-effective treatments. Finally, there is evidence that acamprosate may be associated with reduced lifetime costs due to greater life expectancy that follows the greater abstinence rates achieved while taking acamprosate. ${ }^{48,49}$

Given that acamprosate has been shown to be efficacious, but no more efficacious than alternative medications for alcohol dependence, ${ }^{50}$ an important next step is to examine whether certain individuals are more or less likely to benefit from acamprosate than from other medications. ${ }^{17,51,52}$ Pharmacogenomics has started to reveal that the efficacy of acamprosate may be enhanced by certain genotypes, ${ }^{39}$ although these findings will need to be replicated. Some studies have 
found that individuals with a goal of total abstinence and those patients who are highly motivated may be more likely to benefit from acamprosate than from naltrexone or placebo, ${ }^{37,53}$ while another study found that acamprosate worked better for individuals who were very frequent drinkers prior to starting treatment and less well for those individuals who had more days of abstinence prior to treatment. ${ }^{54}$ Another intriguing finding is that acamprosate does not appear to confer additional benefits when prescribed in combination with behavioral interventions, ${ }^{12}$, although there may be some benefit to providing a motivational intervention prior to starting treatment with acamprosate. ${ }^{53}$

In addition to understanding who will benefit from acamprosate, more research needs to be done on the neurobiological and behavioral mechanisms of change. Recent data suggest that acamprosate may be effective by normalizing dysregulated NMDA-mediated glutamatergic neurotransmission, which may serve to reduce alcohol withdrawalrelated distress and craving. ${ }^{55,56}$ Another study found that acamprosate may be partially effective by reducing the sleep disturbances that are often found among individuals in alcohol withdrawal. ${ }^{31}$ Preclinical studies have found that acamprosate has effects on alcohol self-administration and cue-induced reinstatement of alcohol-seeking, ${ }^{57-59}$ so it has been proposed that acamprosate may work by reducing withdrawal-related and cue-induced craving. ${ }^{18}$ A recent study in alcohol-dependent patients found acamprosateinduced attenuation of craving in response to a priming dose of alcohol and this effect was correlated with the plasma concentration of acamprosate. ${ }^{60}$ Furthermore, individuals who received acamprosate experienced less of an elevation in cortisol levels following alcohol administration, which suggests that acamprosate may play a role in stabilizing hypothalamic-pituitary-adrenal axis functioning. ${ }^{60}$

In summary, acamprosate has been shown to be an effective medication in the treatment of alcohol dependence. However, the effects of acamprosate are only moderate in comparison with placebo and acamprosate has not been found to be consistently superior to naltrexone or disulfiram. Furthermore, there have not been any prospective studies evaluating the long-term (longer than one year) effectiveness of acamprosate in preventing lapse following discontinuation of the medication and retrospective studies have found the long-term effectiveness of acamprosate to be worse than that of disulfiram in a naturalistic clinical setting. ${ }^{61}$ Finally, it is imperative that more research be devoted to studying the mechanisms of efficacy for acamprosate and to determine whether certain patients are more or less likely to respond to this agent, as compared with other FDA-approved medications for alcohol dependence.

\section{Disclosure}

The authors report no conflicts of interest in this work.

\section{References}

1. World Health Organization. Management of substance abuse: Alcohol. 2011. Available at: http://www.who.int/substance_abuse/facts/alcohol/ en/index.html. Accessed November 23, 2011.

2. Hasin DS, Stinson FS, Ogburn E, Grant BF. Prevalence, correlates, disability, and comorbidity of DSM-IV alcohol abuse and dependence in the United States: results from the National Epidemiologic Survey on Alcohol and Related Conditions. Arch Gen Psychiatry. 2007;64: $830-842$.

3. World Health Organization. European Status Report on Alcohol and Health 2010. Copenhagen, Denmark: World Health Organization; 2010 .

4. Koob GF. Alcoholism: allostasis and beyond. Alcohol Clin Exp Res. 2003;27:232-243.

5. Cohen E, Feinn R, Arias A, Kranzler HR. Alcohol treatment utilization: findings from the National Epidemiologic Survey on Alcohol and Related Conditions. Drug Alcohol Depend. 2007;86:214-221.

6. Mark TL, Kranzler HR, Song X. Understanding US addiction physicians' low rate of naltrexone prescription. Drug Alcohol Depend. 2003;71: 219-228.

7. Miller WR, Wilbourne PL. Mesa Grande: A methodological analysis of clinical trials of treatment for alcohol use disorders. Addiction. 2002;9: 265-277.

8. Marlatt GA. Craving for alcohol, loss of control, and relapse: A cognitive-behavioral analysis. In: Nathan PE, Marlatt GA, Loberg T, editors. Alcoholism: New Directions in Research and Treatment. New York: Plenum Press; 1978.

9. Witkiewitz K, Marlatt GA. Modeling the complexity of post-treatment drinking: it's a rocky road to relapse. Clin Psychol Rev. 2007;27: 724-738.

10. Anton RF, O'Malley SS, Ciraulo DA, et al. Combined pharmacotherapies and behavioral interventions for alcohol dependence: The COMBINE study. A randomized controlled trial. JAMA. 2006;29:2003-2017.

11. Witkiewitz K, Maisto SA, Donovan DM. A comparison of methods for estimating change in drinking following alcohol treatment. Alcohol Clin Exp Res. 2010;34:2116-2125.

12. Wolwer W, Frommann N, Janner M, et al. The effects of combined acamprosate and integrative behaviour therapy in the outpatient treatment of alcohol dependence: A randomized controlled trial. Drug Alcohol Depend. 2011;118:417-422.

13. Krampe H, Stawicki S, Wagner T, et al. Follow-up of 180 alcoholic patients for up to 7 years after outpatient treatment: impact of alcohol deterrents on outcome. Alcohol Clin Exp Res. 2006;30:86-95.

14. Rosner S, Hackl-Herrwerth A, Leucht S, Vecchi S, Srisurapanont M, Soyka M. Opioid antagonists for alcohol dependence. Cochrane Database Syst Rev. 2010;12:CD001867.

15. Oslin DW, Berrettini W, Kranzler HR, et al. A functional polymorphism of the mu-opioid receptor gene is associated with naltrexone response in alcohol-dependent patients. Neuropsychopharmacology. 2003;28:1546-1552.

16. Coller JK, Cahill S, Edmonds C, et al. OPRM1 A118G genotype fails to predict the effectiveness of naltrexone treatment for alcohol dependence. Pharmacogenet Genomics. 2011;21:902-905.

17. Mason BJ, Heyser CJ. Acamprosate: a prototypic neuromodulator in the treatment of alcohol dependence. CNS Neurol Disord Drug Targets. 2010;9:23-32.

18. Mann K, Kiefer F, Spanagel R, Littleton J. Acamprosate: recent findings and future research directions. Alcohol Clin Exp Res. 2008;32: 1105-1110. 
19. Pierrefiche O, Daoust M, Naassila M. Biphasic effect of acamprosate on NMDA but not on GABAA receptors in spontaneous rhythmic activity from the isolated neonatal rat respiratory network. Neuropharmacology. 2004; 47:35-45.

20. Daoust M, Legrand E, Gewiss M, et al. Acamprosate modulates synaptosomal GABA transmission in chronically alcoholised rats. Pharmacol Biochem Behav. 1992;41:669-674.

21. Reilly MT, Lobo IA, McCracken LM, et al. Effects of acamprosate on neuronal receptors and ion channels expressed in Xenopus oocytes. Alcohol Clin Exp Res. 2008;32:188-196.

22. Naassila M, Hammoumi S, Legrand E, Durbin P, Daoust M. Mechanism of action of acamprosate. Part I. Characterization of spermidinesensitive acamprosate binding site in rat brain. Alcohol Clin Exp Res. 1998;22:802-809.

23. Rammes G, Mahal B, Putzke J, et al. The anti-craving compound acamprosate acts as a weak NMDA-receptor antagonist, but modulates NMDA-receptor subunit expression similar to memantine and MK-801. Neuropharmacology. 2001;40:749-760.

24. Zornoza T, Cano-Cebrián MJ, Nalda-Molina R, Guerri C, Granero L, Polache A. Assessment and modulation of acamprosate intestinal absorption: comparative studies using in situ, in vitro (CACO-2 cell monolayers) and in vivo models. Eur J Pharm Sci. 2004;22:347-356.

25. Saivin S, Hulot T, Chabac S, Potgieter A, Durbin P, Houin G. Clinical pharmacokinetics of acamprosate. Clin Pharmacokinet. 1998;35: 331-345.

26. Hammarberg A, Beck O, Eksborg S, et al. Acamprosate determinations in plasma and cerebrospinal fluid after multiple dosing measured by liquid chromatography-mass spectroscopy: a pharmacokinetic study in healthy volunteers. Ther Drug Monit. 2010;32:489-496.

27. Mason BJ, Goodman AM, Dixon RM, et al. A pharmacokinetic and pharmacodynamic drug interaction study of acamprosate and naltrexone. Neuropsychopharmacology. 2002;27:596-606.

28. Boothby LA, Doering PL. Acamprosate for the treatment of alcohol dependence. Clin Ther. 2005;27:695-714.

29. Mason BJ, Ownby RL. Acamprosate for the treatment of alcohol dependence: a review of double-blind, placebo-controlled trials. CNS Spectr. 2000;5:58-69.

30. Rubio G, Jimenez-Arriero MA, Ponce G, Palomo T. Naltrexone versus acamprosate: one year follow-up of alcohol dependence treatment. Alcohol Alcohol. 2001;36:419-425.

31. Laaksonen E, Koski-Jannes A, Salaspuro M, Ahtinen H, Alho H. A randomized, multicentre, open-label, comparative trial of disulfiram, naltrexone and acamprosate in the treatment of alcohol dependence. Alcohol Alcohol. 2008;43:53-61.

32. de Sousa A. An open randomized study comparing disulfiram and acamprosate in the treatment of alcohol dependence. Alcohol Alcohol. 2005; $40: 545-548$

33. Kiritze-Topor P, Huas D, Rosenzweig C, Comte S, Paille F, Lehert P. A pragmatic trial of acamprosate in the treatment of alcohol dependence in primary care. Alcohol Alcohol. 2004;39:520-527.

34. Paille FM, Guelfi JD, Perkins AC, Royer RJ, Steru L, Parot P. Double-blind randomized multicentre trial of acamprosate in maintaining abstinence from alcohol. Alcohol Alcohol. 1995;30:239-247.

35. Croissant B, Diehl A, Klein O, et al. A pilot study of oxcarbazepine versus acamprosate in alcohol-dependent patients. Alcohol Clin Exp Res. 2006;30:630-635.

36. Soyka M, Preuss U, Schuetz C. Use of acamprosate and different kinds of psychosocial support in relapse prevention of alcoholism. Results from a non-blind, multicentre study. Drugs R D. 2002;3:1-12.

37. Koeter MW, van den Brink W, Lehert P. Effect of early and late compliance on the effectiveness of acamprosate in the treatment of alcohol dependence. J Subst Abuse Treat. 2010;39:218-226.

38. Kiefer F, Witt SH, Frank J, et al. Involvement of the atrial natriuretic peptide transcription factor GATA4 in alcohol dependence, relapse risk and treatment response to acamprosate. Pharmacogenomics J. 2011;11: $368-374$.
39. Ooteman W, Naassila M, Koeter MW, et al. Predicting the effect of naltrexone and acamprosate in alcohol-dependent patients using genetic indicators. Addict Biol. 2009;14:328-337.

40. Mason BJ, Heyser CJ. The neurobiology, clinical efficacy and safety of acamprosate in the treatment of alcohol dependence. Expert Opin Drug Saf. 2010;9(1):177-188.

41. Bouza C, Angeles M, Munoz A, Amate JM. Efficacy and safety of naltrexone and acamprosate in the treatment of alcohol dependence: a systematic review. Addiction. 2004;99:811-828.

42. Rosenthal RN, Gage A, Perhach JL, Goodman AM. Acamprosate: safety and tolerability in the treatment of alcohol dependence. J Addict Med. 2008;2:40-50.

43. Kennedy WK, Leloux M, Kutscher EC, Price PL, Morstad AE, Carnahan RM. Acamprosate. Expert Opin Drug Metab Toxicol. 2010;6: 363-380.

44. Mason BJ. Acamprosate and naltrexone treatment for alcohol dependence: an evidence-based risk-benefits assessment. Eur Neuropsychopharmacol. 2003;13:469-475.

45. Schwappach D, Popova S, Mohapatra S, Patra J, Godinho A, Rehm J. Strategies for evaluating the economic value of drugs in alcohol dependence treatment. Drug Alcohol Depend. 2011. [Epub ahead of print.]

46. Zarkin GA, Bray JW, Aldridge A, et al. Cost and cost-effectiveness of the COMBINE study in alcohol-dependent patients. Arch Gen Psychiatry. 2008;65:1214-1221.

47. Dunlap LJ, Zarkin GA, Bray JW, et al. Revisiting the cost-effectiveness of the COMBINE study for alcohol dependent patients: the patient perspective. Med Care. 2010;48:306-313.

48. Palmer AJ, Neeser K, Weiss C, Brandt A, Comte S, Fox M. The longterm cost-effectiveness of improving alcohol abstinence with adjuvant acamprosate. Alcohol Alcohol. 2000;35:478-492.

49. Rychlik R, Siedentop H, Pfeil T, Daniel D. Cost-effectiveness of adjuvant treatment with acamprosate in maintaining abstinence in alcohol dependent patients. Eur Addict Res. 2003;9:59-64.

50. De Sousa A. The pharmacotherapy of alcohol dependence: a state of the art review. Mens Sana Monogr. 2010;8:69-82.

51. Kiefer F, Mann K. Acamprosate: how, where, and for whom does it work? Mechanism of action, treatment targets, and individualized therapy. Curr Pharm Des. 2010;16:2098-2102.

52. Wright TM, Myrick H. Acamprosate: a new tool in the battle against alcohol dependence. Neuropsychiatr Dis Treat. 2006;2:445-453.

53. Mason BJ, Goodman AM, Chabac S, Lehert P. Effect of oral acamprosate on abstinence in patients with alcohol dependence in a double-blind, placebo-controlled trial: the role of patient motivation. J Psychiatr Res. 2006;40:383-393.

54. Gueorguieva R, Wu R, Donovan D, et al. Baseline trajectories of heavy drinking and their effects on postrandomization drinking in the COMBINE study: empirically derived predictors of drinking outcomes during treatment. Alcohol. 2011. [Epub ahead of print.]

55. Boeijinga PH, Parot P, Soufflet L, et al. Pharmacodynamic effects of acamprosate on markers of cerebral function in alcohol-dependent subjects administered as pretreatment and during alcohol abstinence. Neuropsychobiology. 2004;50:71-77.

56. Staner L, Boeijinga P, Danel T, et al. Effects of acamprosate on sleep during alcohol withdrawal: A double-blind placebo-controlled polysomnographic study in alcohol-dependent subjects. Alcohol Clin Exp Res. 2006;30:1492-1499.

57. Holter SM, Landgraf R, Zieglgansberger W, Spanagel R. Time course of acamprosate action on operant ethanol self-administration after ethanol deprivation. Alcohol Clin Exp Res. 1997;21:862-868.

58. Spanagel R, Holter SM, Allingham K, Landgraf R, Zieglgansberger W. Acamprosate and alcohol: I. Effects on alcohol intake following alcohol deprivation in the rat. Eur J Pharmacol. 1996;305:39-44.

59. Bachteler D, Economidou D, Danysz W, Ciccocioppo R, Spanagel R. The effects of acamprosate and neramexane on cue-induced reinstatement of ethanol-seeking behavior in rat. Neuropsychopharmacology. 2005;30:1104-1110. 
60. Hammarberg A, Jayaram-Lindstrom N, Beck O, Franck J, Reid MS. The effects of acamprosate on alcohol-cue reactivity and alcohol priming in dependent patients: a randomized controlled trial. Psychopharmacology. 2009;205:53-62.

61. Diehl A, Ulmer L, Mutschler J, et al. Why is disulfiram superior to acamprosate in the routine clinical setting? A retrospective long-term study in 353 alcohol-dependent patients. Alcohol Alcohol. 2010;45: 271-277.
62. Kampman KM, Pettinati HM, Lynch KG, et al. Initiating acamprosate within-detoxification versus post-detoxification in the treatment of alcohol dependence. Addict Behav. 2009;34:581-586.

63. Pelc I, Ansoms C, Lehert P, et al. The European NEAT program: an integrated approach using acamprosate and psychosocial support for the prevention of relapse in alcohol-dependent patients with a statistical modeling of therapy success prediction. Alcohol Clin Exp Res. 2002;26:1529-1538

Therapeutics and Clinical Risk Management

\section{Publish your work in this journal}

Therapeutics and Clinical Risk Management is an international, peerreviewed journal of clinical therapeutics and risk management, focusing on concise rapid reporting of clinical studies in all therapeutic areas, outcomes, safety, and programs for the effective, safe, and sustained use of medicines. This journal is indexed on PubMed Central, CAS
EMBase, Scopus and the Elsevier Bibliographic databases. The manuscript management system is completely online and includes a very quick and fair peer-review system, which is all easy to use. Visit http://www.dovepress.com/testimonials.php to read real quotes from published authors.

Submit your manuscript here: http://www.dovepress.com/therapeutics-and-clinical-risk-management-journal 